\title{
FoodsE-KINDEX score is associated with reduced blood pressure levels in children: the CYKIDS Study
}

\author{
Chrystalleni Lazarou, Demosthenes Panagiotakos and Antonia-Leda Matalas \\ Harokopio University, Athens, Greece
}

The most appealing approaches for the reduction of blood pressure (BP) levels are those that involve dietary modifications ${ }^{(1)}$. For children there are few studies that have examined the relationship between dietary patterns and BP levels, and they have reported conflicting results $^{(2)}$

The aim of the present study was to investigate the association between dietary habits and BP levels in children. Data from a subsample of the CYKIDS national cross-sectional study consisting of 634 children (11.7 (SD 0.83) years) was studied ${ }^{(3,4)}$. Measurements included BP, height, weight and waist circumference (WC). BMI was calculated according to International Obesity Taskforce criteria ${ }^{(5)}$. Dietary patterns and quality were assessed by the FoodsE-KINDEX score ${ }^{(4)}$. Table 1 presents the components of FoodsE-KINDEX.

\begin{tabular}{lccccc}
\multicolumn{6}{l}{ Table 1. FoodsE-KINDEX components, along with the scoring system, the upper and lower cut-off points of each component* } \\
\hline Response categories........ & Never & Sometimes & Often & Very often \\
No. of times per week.... & 0 & $1-2$ & $3-5$ & $\geq 6$ & Range of score \\
\hline Bread & 0 & 1 & 1 & 3 & $0-3$ \\
Cereals and grain foods (excluding bread) & 0 & 1 & 2 & 2 & $0-2$ \\
Fruit and fruit juices & 0 & 1 & 2 & 3 & $0-3$ \\
Vegetables & 0 & 1 & 2 & 3 & $0-3$ \\
Legumes & 0 & 1 & 3 & 3 & $0-3$ \\
Milk & 0 & 1 & 2 & 2 & $0-3$ \\
Fish and seafood & 0 & 3 & 3 & 0 & $0-3$ \\
Meat & 1 & 3 & 0 & $0-3$ \\
Salted and smoked meat food & 3 & 1 & 1 & $0-3$ \\
Sweets and snack items & 2 & 2 & 1 & 0 & $0-2$ \\
Soft drinks & 3 & 3 & 1 & 0 & $0-3$ \\
Fried food & 2 & 3 & 3 & 2 & $0-3$ \\
Grilled food & 0 & 3 & & $0-3$ \\
\hline
\end{tabular}

*Based on consumption frequency (total possible range of score 0-37).

Binary and multinomial logistic regression analyses were applied with categorical dependent variables, systolic BP (SBP), diastolic $\mathrm{BP}$ (DBP) and BP levels, and independent variables, the two categories of the FoodsE-KINDEX score (below $v$. above median FoodsE-KINDEX score), after controlling for potential confounders (BMI, WC, age, gender, socio-economic status, physical activity, television viewing).

Children with FoodsE-KINDEX scores above the median (61\%; OR 0.39 (95\% CI 0.18, 0.85)) were less likely to have elevated SBP levels (i.e. $>120 \mathrm{mmHg}$ ) compared with children with a score below the median; however, a non-significant association was observed for DBP levels (OR 0.47 (95\% CI 0.18, 1.25)). Furthermore, the multinomial logistic analysis revealed that children with low FoodsEKINDEX scores were 6.6 times more likely to have elevated levels for both SBP and DPB (>120/80 mmHg) compared with those with high FoodsE-KINDEX scores (95\% CI 1.13, 39.27).

Healthy dietary habits were independently associated with lower BP levels among healthy children. This finding may have implications for public health and should be further explored.

This work was supported by a Cyprus Research Promotion Foundation research grant (AKGEN/0506/05). The study was also partially supported by the 'Charalambides' dairies and the Cyprus Dietetic Association. We would like to sincerely thank the participating children and their parents. Finally, we would like to thank the Cyprus Ministry of Education \& Culture (Department of Primary Education) and all the teachers who readily consented to carry out the study during school hours.

1. Sacks FM, Svetkey LP, Vollmer WM et al. (2001) N Engl J Med 344, 3-10.

2. Couch SC \& Daniels SR (2005) Curr Opin Pediatr 17, 642-6647.

3. Lazarou C, Panagiotakos DB, Panayiotou G \& Matalas A-L (2008) Obes Rev 9, 185-193.

4. Lazarou C, Panagiotakos DB \& Matalas A-L (2008) Int J Obes (Lond) 32, Suppl. 1, S216.

5. Cole TJ, Bellizzi MC, Flegal KM \& Dietz WH (2000) BMJ 320, 1240-3. 